\title{
Neuron-less knowledge processing in forests
}

Dr Aviv Segev, Professor
at the University of South Alabama, is researching the knowledge-related activity of forests. While trees do not have neurons, they can build a communication network, use of the reson use of the resources $n$ demonstrates that neuro like relations occur in a forest knowledge-processing system. Results demonstrate that trees use mechanisms analogous to neural network knowledge processing, and communicating knowledge in forests resembles the communication between trying to optimise a decision.

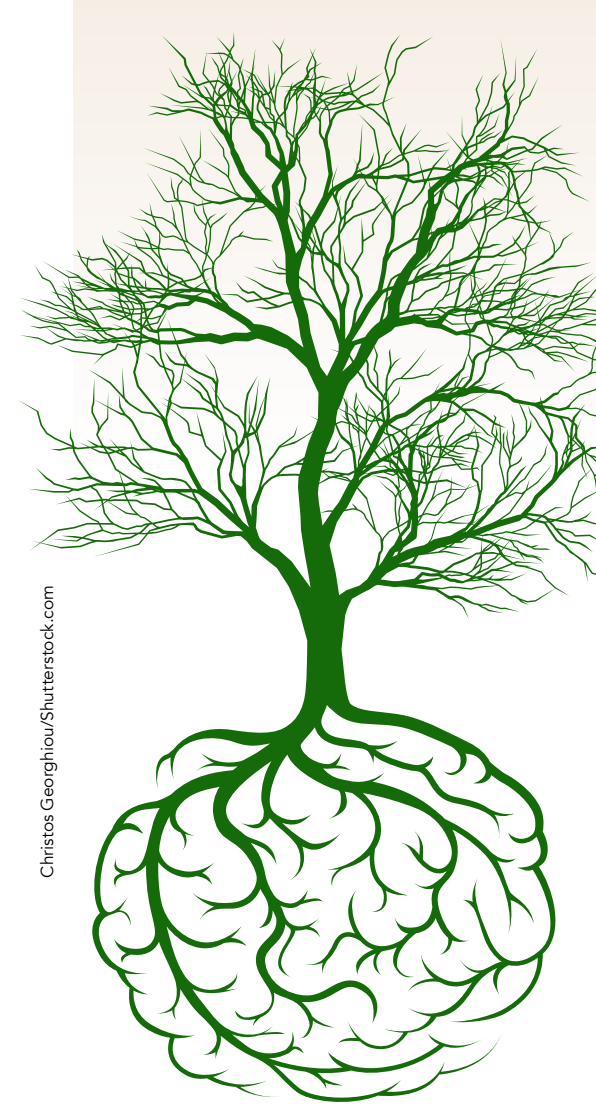

their attempts to optimise the use of sunlight. This study was inspired neurons is removed from the brain to enable the neurons in the surrounding area to become less active and resume normal neuron activity. To emulate this procedure, Dr Segev examined areas of forest where sections of trees had been removed to form trails.

\section{ANALYSIS}

ch team analysed 323 forests in 19 different national forest locations across the US. ArcGIS, a spatial analysis tool, was used to calculate canopy coverage and distance from trails using deterministic and geo-statistical techniques. The researchers sampled 2802 trails in these forests and analysed the degradation and regeneration the surrounding areas, up to $450 \mathrm{~m}$ from each trail. The behaviour of the trees on both sides of the trails was observed, together with the change in the use of light as the distance from the trails increased.

This analysis revealed that, as with the surrounding neurons in an epilepsy resection, these forest resections cause nearby trees to become less active, and thereby less efficient at processing knowledge. Dr Segev explains that, while resection in the brain results in hyper-acting neurons resuming normal activity, in trees it 'falls down to abnormal activity'.

\section{A FOREST KNOWLEDGE}

\section{PROCESSING SYSTEM}

Overall, the forest canopy was found to increase logarithmically as the

distance from the resection increased. This suggests that, after resection, the trees attempt to form independent entities, with each part of the forest processing network knowledge their access to light. The researchers observed some exceptions to this pattern, with forest structures similar to the neuron semi-sphere structures that occur in brain tumours, demonstrating that neuron-like relations can occur in forest knowledge-processing system.

A manual analysis of those cases where the forests' canopy coverage
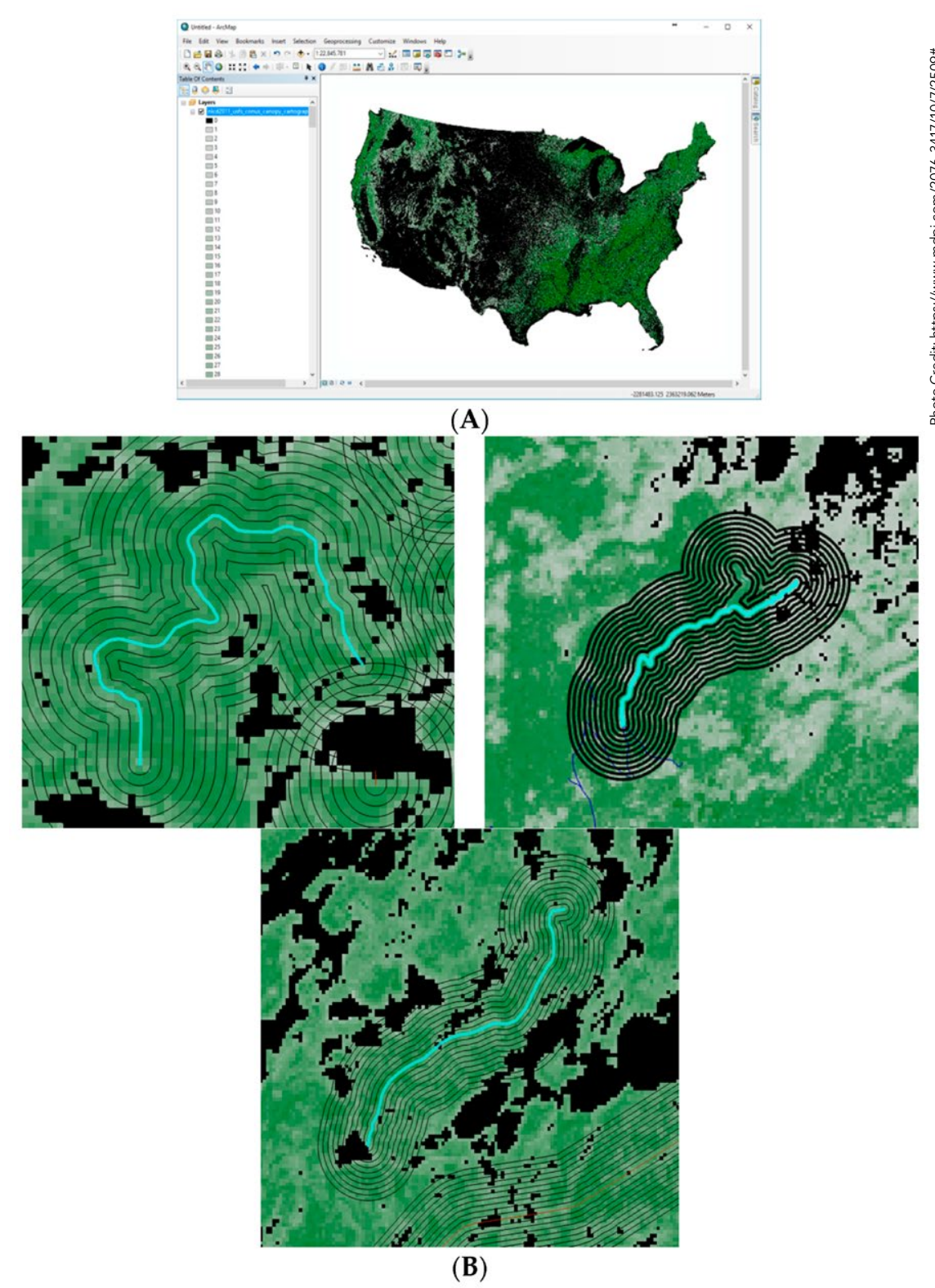

Figure 1: Tree canopy across the US (A). Tree canopy coverage surrounding forest trails (B). The
light blue-green line is the actual trail . The black rinys surrounding the trail reperesent thinreasing distances from the trail center, 45 mapart. The green map layout represents canopy coverage per location, image pixel- $-30 \mathrm{~m}^{2}$, calculated based on satellite imagery. The darker the shade of
green, the greater the canopy coverage. For each ring extending the distance around the trail, the

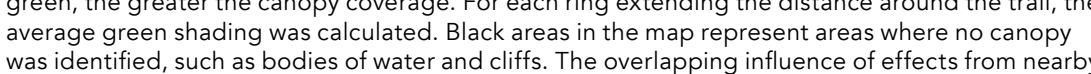
was identified, such as bodies of water and diliff. The overlapping influence o o effectss
trails was also analysed, as can be seen from other coinciding rings from other trails.

Viewing a forest as a network helps to explain the benefit of communication for resource optimisation.

did not increase with increasing distance from the trails, uncovered several explanations. The most common reason involved large bodies to the trail. Oth lakes or rivers, close to the trall. Other resasons included for agriculture, residences, and roads drop in height was an unfavourable evironment for tree canopies.

The results also show that the forest canopies were affected by logging 

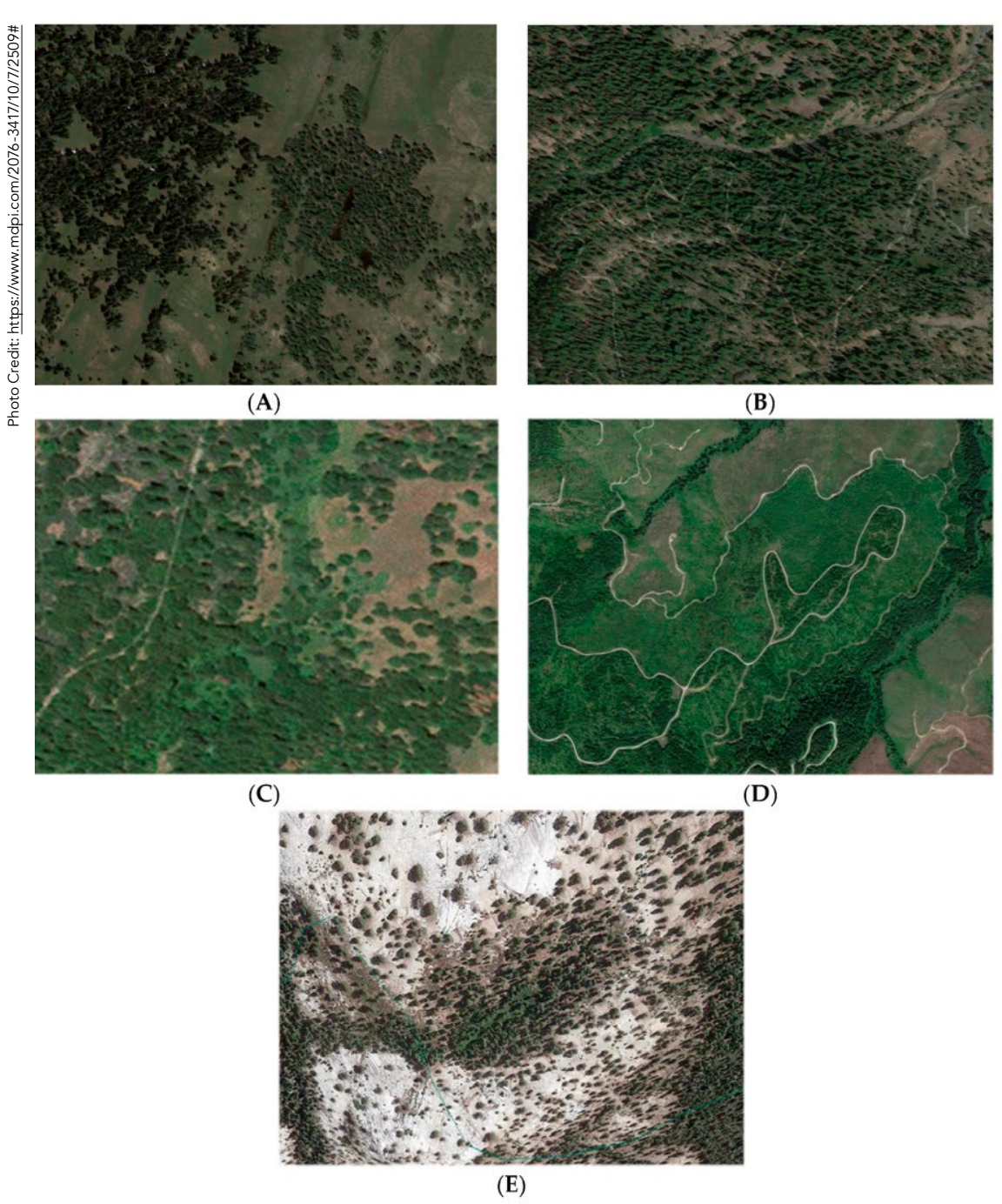

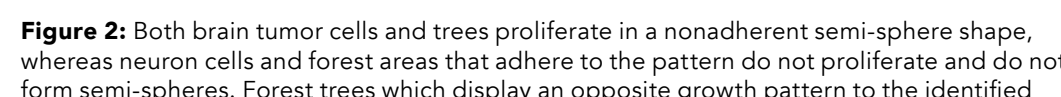
whereas neuron cels.
form semi-shperes. Fort trees which display an opposite growth pattern to the identified
tree formation in: Bridger Teton National Forest (A), Okanogan-Wenatchee National Forest

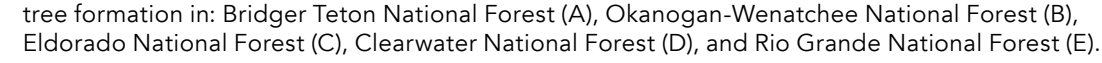

The network between trees prioritises the resource optimisation of the forest over that of a single tree.

and they offer an explanation for the increased damage as well as the regrowth pattern in both the natural and logging gaps. Examining canopy damage and ground disturbance in skid trails used by logging equipm to remove logs and tree-fall gaps showed that the damage can be
much deeper than shown in previous much deeper than shown in previous recovery process is similar to the

recovery of a neural network from an error value, a process known as 'readjusting the weights' of the neur network.

\section{SIMILAR KNOWLEDGE} TRANSMISSION The network structure and behaviour demonstrate the similarity between forest trees and human neurons in have similar abilities enabling them to process knowledge concerning optimise their use. Moreover, just as neuron prioritises the resource as as of the network over its own needs, this research demonstrates that the network between trees prioritises the resource optimisation of the forest over that of a single tree. These results show that the communication of knowledge in forests is analogous to the communication between neurons in the brain when trying to optimise a decision.

This research highlights how the optimisation of canopy coverage over the entire forest arises from a proces of shared knowledge among all the of the network is transmitted, and results in the entire network trying overcome loss and reoptimise the use of resources.

An important aspect of this research is that it facilitates the analysis of neuron-like behaviour in an environment that processes knowledge far slower than human neurons. While the nature-inspired knowledge processing demonstrated in the forest environment has similar characteristics to brain neuron processing, it is easier to manipulate and control, making it a potential vehicle for understanding and solving a variety of problems

\section{BROADER IMPLICATIONS}

This research begs an important philosophical question: how unique is knowledge processing in the processing frequently appear in nature? The neural-network processing by trees revealed by this research may be just one example of knowledgeprocessing behaviour in nature; other systems may exist where components other than neurons perform basic knowledge processing for resource usage. Dr Segev remarks that 'other neural network knowledge-processing models might be common, and "which we call "thinking" mocessing which we cal "thinkng" might be widely used by processing mechanisms

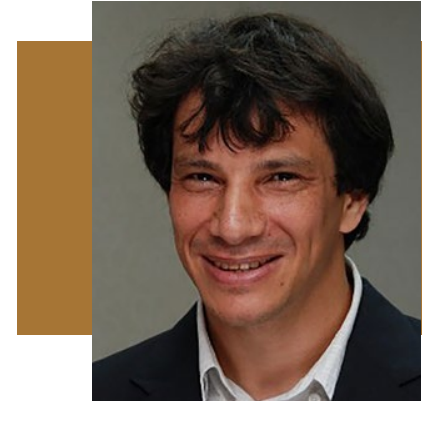

\section{Behind the Research} Dr Aviv Segev

E: segev@southalabama.edu T: +12514607975 W: http://schoolofcomputing.southalabama.edu/ segev

\section{Research Objectives}

Dr Aviv Segev is researching communication and ds it ana ons to a neural network.

\section{Detail}

Aviv Segev

Mobile, Alaberices Drive

Bio

Aviv Segev spends his time looking for the DNA of knowledge, an underlying structure common to all knowledge, through analysis of knowledge models in natural sciences, knowledge processing in natural and artificial neural networks, and knowledge mapping between different knowledge domains. Find him at University of South Alabama, Computer Science Department.

Collaborators - Dorothy Curtis (M - Christine Balil

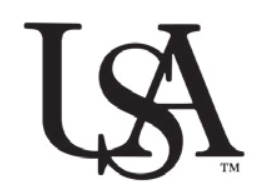

UNIVERSITY OF SOUTH ALABAMA

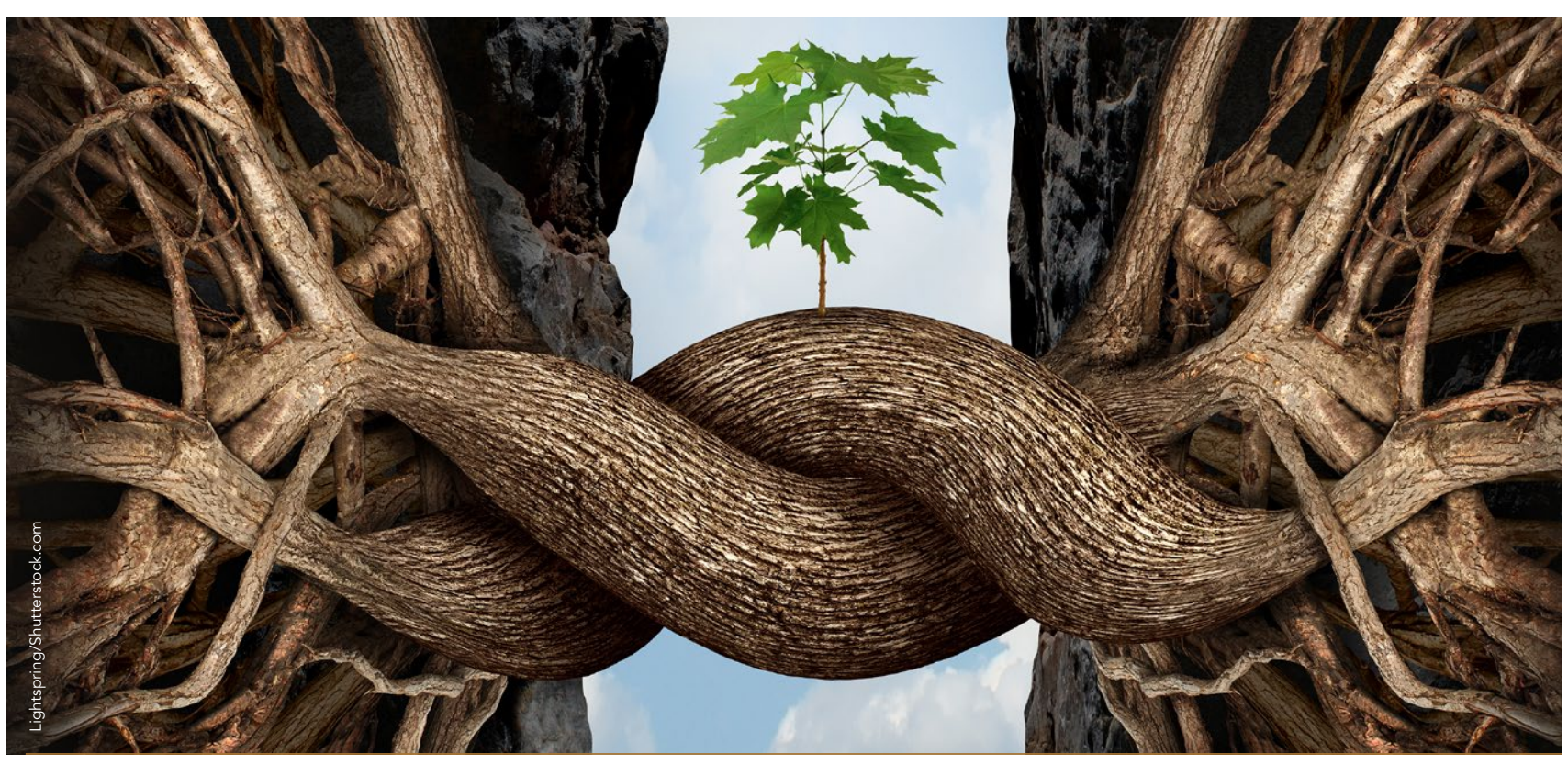

\section{References}

Segev, A., Curtis, D., Balili C. \& Jung, S. (2020). Neuronless Knowledge Processing in Forests. Applied Sciences app10072509

Segev, A., Curtis, D., Jung, S. \& Chae, S. (2016). Invisible Brain: Knowledge in Research Works and Neuron Activity doi.org/10.1371/journal.pone. 0158590

\section{Personal Response}

What prompted you to study the forest as a system rather than examining the behaviour of individual

II Looking for an underlying model for knowledge which expands across domains, you want to identify a system most people agree can process knowledge, Analysing individual trees is like analysing different biological systems instead of similar ones. Furthermore, the idea of analysing a single tree as a system that was suggested by Darwin. So far botanists haurns ib to to in an individual tree. This is why lapproached the issue from a computer science perspective. [online], 10(7), 2509. Available at: https://doi.org/10.3390/ similar components and show it processes information. 